\title{
METODOLOGÍAS DE PLANIFICACIÓN DEL USO PÚBLICO EN ESPACIOS PROTEGIDOS: ANTECEDENTES Y PERSPECTIVAS FUTURAS
}

\author{
Juan Pablo Morea \\ Departamento de Geografía, Universidad Nacional de Mar del Plata y CONICET (Argentina)
}

\begin{abstract}
RESUMEN
Los espacios protegidos se enfrentan en la actualidad a grandes problemáticas territoriales. Diversos estudios han demostrado que la efectividad de su manejo presenta importantes deficiencias asociadas a la imposibilidad de compatibilizar actividades de uso y conservación, y en los últimos años ha crecido el interés por planificar el uso público como estrategia para mejorar el manejo de estos espacios. Mediante una extensa revisión bibliográfica de experiencias en distintos países, este trabajo se propuso relevar distintas experiencias recientes para identificar los factores clave de este tipo de procesos, pero también las principales carencias evidenciadas.

Las distintas visiones y aportes de las experiencias relevadas contribuyen a la construcción de una perspectiva metodológica sustentada en base a una serie de componentes clave para la planificación del uso público en estos espacios. De forma complementaria, a partir de los resultados de este estudio se han podido identificar perspectivas de planificación que podrían guiar iniciativas de esta índole en el futuro próximo.
\end{abstract}

Palabras clave: Espacios protegidos; planificación; uso público; ordenamiento territorial; metodologías.

\section{PLANNING METHODOLOGIES OF THE PUBLIC USE OF PROTECTED AREAS: BACKGROUND AND FUTURE PROSPECTS.}

\section{ABSTRACT}

The protected spaces are currently facing major territorial issues. Various studies have shown that the effectiveness of its management presents important deficiencies associated with the impossibility of reconciling activities of use and conservation, and in recent years has increased the interest in public use planning as a strategy to improve the management of these spaces. Through an extensive literature review of experiences in different countries, this work is proposed to relieve various recent experiences to identify the key factors of this type of processes, but also the main shortcomings highlighted.

The various visions and contributions of the experiences surveyed, contribute to the construction of a methodological perspective based on a number of key components for the planning of public use in these spaces. In a complementary way, on the basis of the results of this study, have been able to identify prospects of planning which could guide initiatives of this nature in the near future.

Keywords: Protected areas; planning; public use; land use planning; methodologies.

Universidad Nacional de Mar del Plata, Departamento de Geografía (Mar del Plata, Argentina). E-mails: juanpablomorea@gmail.com

Fecha de recepción: 31 de mayo de 2016. Fecha de aceptación: 06 de octubre de 2016. 


\section{INTRODUCCIÓN}

Los espacios protegidos (EP) se erigen como un espacio más que se encuentra inmerso en los grandes conflictos y problemáticas territoriales que afrontan la mayor parte de los países del mundo en la actualidad. Estos espacios que en sus inicios fueron creados por su alto valor para la conservación de la diversidad biológica y para la preservación de herencias culturales y bellezas escénicas, ya no pueden ser vistos como grandes extensiones territoriales aisladas e independientes.

Desde hace varias décadas los criterios para la creación y diseño de EP se han ido complejizando y ha sido creciente la preocupación por optimizar su gestión y manejo. El crecimiento de la población y la expansión de las fronteras urbanas y productivas han generado una gran competencia por el espacio que se ha convertido en una de las principales amenazas para la conservación de la biodiversidad.

Una tendencia creciente ha manifestado su preocupación por la viabilidad a largo plazo de las unidades de conservación. Se cree que los EP tendrían mayores posibilidades de contribuir a la conservación de la biodiversidad si formaran parte de un sistema representativo de reservas; de una red. Por eso, la discusión no sólo pasa por la elección de un sitio para crear un EP o por el tamaño que el mismo debiera tener; sino también por el modo que la combinación de diversas áreas puede conformar un conjunto representativo de reservas, incluso pensando a nivel global.

No obstante ello, es imprescindible para el funcionamiento de un sistema de espacios protegidos que exista un adecuado manejo individual de cada unidad. La preocupación por la efectividad en el manejo y la gestión de EP ha propiciado la proliferación de numerosas metodologías para evaluarlas, fundamentalmente derivadas de acuerdos internacionales como el Convenio Sobre la Diversidad Biológica, e impulsadas por organismos especializados en la temática como la Unión Mundial para la Conservación de la Naturaleza (UICN) o el Fondo Mundial para la Naturaleza (WWF).

Cuantiosos estudios han sido desarrollados y replicados en una gran cantidad de países a raíz de dicha preocupación (ALMIRÓN et al., 2007; BURKART et al., 2005; ELBERS, 2011; GIACCARDI et al., 2007; GÓMEZ MENDOZA et al., 1999), concluyendo generalmente que el estado actual de conservación de estos importantes espacios es muy deficiente.

Cada EP tiene un contexto propio, que lo diferencia del resto y que, en muchos casos, no permiten que se hagan generalizaciones acerca de los problemas que los atañen. Sin embargo, es sabida la dificultad que representa gestionar territorios tan complejos y existen desafíos comunes a todos los espacios. Las falencias más importantes que pueden encontrarse en todos estos estudios giran en torno a tres ejes principales: la debilidad institucional, la falta de un ordenamiento territorial y la ausencia de planificación del uso público (MOREA, 2014).

Los criterios de planificación históricos de estos espacios han estado fuertemente vinculados a los conceptos de la biología de la conservación y a disciplinas más preocupadas por factores naturales que humanos. Las actividades de uso público se han desarrollado en muchos casos sin planificación alguna y en ocasiones de forma irregular, sin el involucramiento de especialistas en la materia, o con visiones parciales y sesgadas. En el contexto actual, los EP no pueden ser entendidos sin la presencia de las actividades humanas, y en este punto es donde la correcta articulación del binomio uso-conservación deviene fundamental para alcanzar las metas preestablecidas por cada reserva.

El interrogante que surge de este análisis es si podremos lograr compatibilizar la conservación con las actividades de uso público. Hasta el momento esta tarea ha sido dificultosa, pero está claro que sin una correcta estrategia de planificación y ordenación de estas actividades, será imposible. Por este motivo, se propuso como objetivo central de este trabajo, analizar las principales metodologías y técnicas de los últimos años referidas a la planificación del uso público en espacios protegidos. Complementariamente, se realizó una propuesta metodológica para el abordaje de esta complicada tarea, enmarcada en la situación actual y con vistas a los desafíos de los próximos años. 
METODOLOGÍAS DE PLANIFICACIÓN...

\subsection{MÉTODO DE TRABAJO}

Para alcanzar los objetivos planteados se propone un abordaje crítico analítico basado en una revisión bibliográfica en busca de experiencias de distintos países y documentos de organismos internacionales especializados en la problemática. La elección de esta perspectiva responde, en primer lugar, a la existencia de una importante cantidad de documentos inherente a la temática, pero fundamentalmente a la convicción de que la mirada analítica permite revelar factores no observados previamente y proceder a identificar su significado a la luz de un contexto específico propio.

Realizar un análisis global de la cuestión resulta dificultoso debido a la cuantiosa cantidad de experiencias y países involucrados; no obstante ello, se consideraron en este trabajo informes de países referentes de América, Europa y Asia. Entre ellos, pueden mencionarse los casos de Argentina, Brasil, Canadá, China, Colombia, Costa Rica, España, Estados Unidos e Italia, entre otros. A su vez, el análisis de documentos e informes realizados por organismos como la UNESCO, la UICN, la OEA, la FAO, la WWF, o EUROPARC otorgan una perspectiva ampliada y reflejan una situación integral respecto a la conservación de la biodiversidad en la escala planetaria.

El trabajo se estructuró sobre la base de tres etapas o fases. En la primera de ellas el relevamiento estuvo dirigido a la identificación de las principales problemáticas que enfrentan estos espacios, en vistas de la histórica relación sociedad naturaleza y su manifestación conflictiva o disfuncional. Este primer análisis impulsa en un segundo momento al reconocimiento de la voluntad de subsanar las problemáticas de estos espacios para mejorar su gestión y manejo; y a la identificación de las principales propuestas metodológicas y al conjunto de técnicas e instrumentos de planificación derivados de esta voluntad. Complementariamente se realizó un análisis de experiencias concretas en distintos países para identificar factores clave o patrones comunes entre las distintas propuestas metodológicas. El objetivo de esta fase responde a la necesidad de encontrar criterios y perspectivas comunes en esta asignatura, pero fundamentalmente a la intención de identificar falencias que permitieran elaborar las bases para una nueva construcción metodológica.

La última etapa consistió en elaborar una propuesta metodológica que combine los aspectos más destacados de las distintas experiencias analizadas. La confección de esta propuesta se erigió sobre el criterio de reunir las condiciones necesarias para su posible aplicación, pero a la vez pensando en poner de manifiesto los principales ejes que marcarán las perspectivas futuras de planificación del uso público en espacios protegidos.

\section{LA COMPETENCIA POR EL ESPACIO Y LA NECESIDAD DE UN ORDENAMIENTO TERRITORIAL}

Espacio y sociedad poseen un vínculo histórico y una relación dinámica que ha ido evolucionando y mutando con el paso del tiempo. En esta relación el hombre ha desempeñado un rol dominante de transformación y de ordenación. El rol desarrollado por el hombre, planteaba ISNARD (1975), se ha ido complejizando en la medida en que las sociedades evolucionaron y desarrollaron una gran cantidad de medios para volver cada vez más funcional el espacio a las expectativas y deseos humanos. Con el advenimiento de las sociedades modernas y la incorporación de los medios de capital financiero, la búsqueda y conquista de nuevos espacios se extendió de forma tal que la escasez de nuevos territorios se ha vuelto una característica de los tiempos actuales.

La visión de los espacios naturales, vírgenes y prístinos está cada vez más alejada de la realidad y según SANTOS (1997) no hay hoy una separación entre hombre y naturaleza ya que el hombre está presente y ha conquistado todos los espacios naturales, aunque no les haya otorgado un uso específico todavía. Los cambios en la configuración y en la organización del territorio y las nuevas formas de producción obligan a renovar los enfoques del pasado. Siguiendo esta noción, nos referimos en este trabajo a los espacios protegidos no como espacios naturales, sino como espacios sociales, pensados y construidos por el hombre para la conservación de valores primordialmente naturales, pero también sociales y culturales.

Papeles de Geografía, 62 (2016), 119-136 
Justamente en base a la idea de la existencia de una producción del espacio es que SANTOS (1997) justifica la necesidad de nuevos enfoques en la geografía. Enfoques que deben orientarse a regular ciertas formas de aprovechamiento del espacio. El mercado habitualmente no regula lo suficiente la producción del espacio ni el acceso del capital a la naturaleza. La sobreexplotación y la inercia de expansión del capitalismo, llevan a la búsqueda constante de nuevos espacios y al abandono de tierras degradadas para su descarte o refuncionalización.

La producción del espacio bajo estas condiciones, se constituye en la principal amenaza de los EP. Esta expansión desregularizada del capital genera una fuerte competencia por el espacio, que lleva indefectiblemente a la aparición de incompatibilidades en el uso del suelo. Los EP se ven cercados por la expansión de las ciudades, de la frontera agraria, de la deforestación, de la explotación minera y, en definitiva, de cualquier otro tipo de utilización que sea rentable en términos económicos. La distancia entre los EP y los centro urbanos más cercanos se va reduciendo y se estima que para 2030 una gran parte de las reservas del mundo estarán a tan solo media hora en auto de las ciudades más próximas (MCDONALD et al., 2008; MCDONALD et al., 2009).

La problemática territorial manifiesta en las líneas anteriores refleja la relación que las reservas mantienen con su entorno. Pero también existe una problemática interna, porque las reservas necesitan ordenar los usos y actividades que en ellas se desarrollan. El uso público en los EP está habitualmente relacionado con la actividad turística. Si bien es cierto que el turismo está presente en casi todos los casos y que suele ser la principal actividad en este tipo de espacios, bajo la denominación del uso público pueden comprenderse muchas otras actividades. En la actualidad podemos encontrar EP donde además de las actividades de conservación, investigación y educación ambiental, conviven usos turísticos, urbanos, comerciales, pesqueros y hasta industriales.

Queda claro ante esta situación, que los EP no escapan a las problemáticas territoriales existentes, fundamentalmente articuladas en torno a la tenencia de las tierras y los conflictos por incompatibilidades de uso. Estos son los principales motivos que nos llevan a una urgente necesidad de establecer un ordenamiento territorial que permita frenar esta expansión autodestructiva y armonizar la relación entre los usos internos en cada unidad de conservación.

La crisis ambiental en la que hoy estamos inmersos ha llevado al Estado a ejercer mayor control sobre las formas de producción del espacio, y esta necesidad de control es la que dispara una multitud de nuevos instrumentos de planeación; más flexibles, más sociales y más participativos. El ordenamiento territorial representa un determinado estilo de planificación, que se ha ido conformando con el transcurrir de los años y de muchas experiencias. Posee características propias que hoy nos permiten hablar de sus valores intrínsecos como concepto y como instrumento de planificación, pero no puede desprenderse de las influencias de las distintas corrientes y tendencias dentro de la planificación.

Algunos autores consideran que el ordenamiento territorial es precisamente la evolución de los distintos métodos de planificación ligados al territorio, como la planificación regional, el urbanismo, la planificación económica y la ambiental (MASSIRIS CABEZA, 2002; GUDIÑO, 2010). Según esta visión el ordenamiento territorial debiera representar entonces una combinación de los mejores aportes de cada uno de estos estilos de planificación y conformar una visión superadora. El cambio fundamental que se plantea viene dado por la flexibilización de los procesos de planificación, por la integración de múltiples disciplinas y campos de conocimientos y por el desarrollo de programas de participación ciudadana.

Sin pretender en este trabajo hacer un repaso de las numerosas definiciones e interpretaciones de este concepto, se entiende que el ordenamiento territorial debiera presentarse como una alternativa llamada a saldar las deficiencias de experiencias anteriores en planificación. La necesidad de ordenar un territorio, y en particular los EP, está referida a la construcción de una relación sociedadnaturaleza que permita alcanzar los objetivos de conservación y satisfacer las necesidades e intereses de las comunidades involucradas.

En ese sentido, los objetivos principales de una propuesta de estas características deberán relacionarse con: propiciar un equilibrio territorial a todas las escalas; garantizar la equidad social 
METODOLOGÍAS DE PLANIFICACIÓN...

en todas las dimensiones; establecer principios de sustentabilidad que sean ejes articuladores de los planes; desarrollar mecanismos de participación social que realmente sean representativos, que tengan influencia en la toma de decisiones y que estén presentes a lo largo de todo el proceso de planificación y gestión; lograr generar espacios más habitables y de calidad, tanto en entornos urbanos como en zonas rurales y naturales; optimizar los mecanismos de gestión; propiciar una planificación orientada a la acción y generar procesos mucho más versátiles y dinámicos.

Un concepto muy vinculado al ordenamiento territorial dentro de los EP es el de zonificación. Esencialmente zonificar consiste en establecer una subdivisión interna de carácter funcional que ordena el uso del espacio. La zonificación se presenta como una herramienta fundamental para el manejo de un EP cuya finalidad es orientar, distribuir y regular los usos y actividades admitidas en el área según su categoría de manejo y sus objetivos. El desafío primordial consiste en establecer estrategias de uso del suelo y aprovechamiento de los recursos que se integren a las estrategias de conservación.

Las zonificaciones y los diseños que se han implementado en este tipo de espacios se han caracterizado por una excesiva orientación hacia los aspectos físico-naturales y no han tenido demasiado en cuenta a los aspectos sociales. Existe una ausencia de planificación del turismo y de otras actividades o usos productivos, o en muchos casos estos procesos de planificación se llevan a cabo de forma aislada y sin integrarse en los Planes de Manejo o en los planes operativos de cada parque.

No puede desconocerse que se produjeron en los últimos años diversas iniciativas y estudios que se han centrado en estudiar y reevaluar los criterios de planificación y zonificación adoptados. No obstante, como consecuencia de las tendencias mencionadas, se evidencian aún grandes disfuncionalidades e incompatibilidades de usos que no permiten la consecución de los principales objetivos planteados por cada reserva.

En este contexto de competencia por el espacio, difícilmente podamos imaginar EP enteramente dedicados a las funciones de conservación y preservación. Cada vez más se requiere su multifuncionalidad y su integración con los intereses y las actividades de las comunidades cercanas. La adaptación de los EP a ciertas prácticas económicas de bajo impacto podría resultar una estrategia de disminución de los conflictos sociales, permitiendo su subsistencia a largo plazo. Una estrategia que podría evitar la colonización de estos espacios por los intereses de las megas explotaciones o especulaciones inmobiliarias.

Por eso, la planificación del uso público no puede ser subvalorada y debe entenderse como una herramienta fundamental y estratégica para lograr la compatibilización entre las actividades de uso y conservación. No obstante, para lograrlo será necesario establecer un nuevo marco conceptual y metodológico inspirado en una concepción amplia e innovadora del ordenamiento territorial que redefina los modelos de zonificación y establezca nuevas técnicas e instrumentos para llevar adelante la planificación y el manejo de los EP.

\section{METOdOLOGÍAS DE PLANIFICACIÓN DEL USO PÚBLICO EN ESPACIOS PROTEGIDOS}

La planificación del uso público no ha sido una prioridad en el manejo de los EP. En general las decisiones respecto a la creación, el diseño, el tamaño y la gestión de estos espacios han estado dominadas por los criterios de la biogeografía, la biología de la conservación y las ciencias naturales en general. El objetivo siempre estuvo centrado en las necesidades de las especies a proteger y los procesos de planificación se han focalizado en diseñar estrategias a tal fin.

A pesar de ello, los EP históricamente han convivido con otros usos y actividades que generan impactos negativos y que dificultan el alcance de los objetivos de conservación. Estos problemas podemos atribuirlos a la ausencia de planificación del uso público o a la menor importancia atribuida en relación a los objetivos de conservación. Pero las deficiencias en la planificación no sólo pueden asociarse al uso público; son una problemática general a nivel mundial. 
En los últimos años diversos trabajos se han realizado para evaluar el estado de la gestión los EP. En Latinoamérica algunos estudios importantes que pueden destacarse son "Estado Actual de las Áreas Naturales Protegidas de América Latina y el Caribe" (DE LA MAZA ELVIRA et al., 2003), "Gestión de áreas protegidas para la conservación de la biodiversidad: evidencias de Brasil, Honduras y Perú" (DOUROJEANNI et al., 2006), "Las Áreas Protegidas de América Latina: Situación actual y perspectivas para el futuro" (ELBERS, 2011) o las recomendaciones del segundo Congreso Latinoamericano de Parque Nacionales y otras Áreas Protegidas.

Las conclusiones de estos trabajos reflejan ausencia o desactualización de planes de manejo y de planes operativos anuales en la mayoría de los EP de cada país. En algunos casos como en Argentina, Uruguay, Bolivia y Perú sólo entre el 20 y el 30\% de los EP poseen planes vigentes; y los países con mayor desarrollo como Costa Rica, Chile o Brasil registran valores entre el 40 y el 50\%. En Europa los documentos elaborados por Europarc manifiestan una preocupación por problemáticas similares. En España por ejemplo, según el Anuario 2013 de esta federación sólo el $12 \%$ de los hábitats puede considerarse en un estado de conservación favorable (EUROPARC, 2014).

Lo cierto, es que más allá de experiencias exitosas y del progreso y avance de algunos países que poseen una larga tradición en conservación del medio ambiente y la biodiversidad, la preocupación por mejorar el estado de la gestión de los EP es una tendencia a nivel mundial. La falta de eficacia en el alcance de los objetivos de conservación es lo que ha motivado la búsqueda de estrategias complementarias a las tradicionalmente predominantes. En los últimos 20 años la preocupación por ordenar las actividades de uso público dentro de estos espacios ha cobrado un gran ímpetu; entendiendo que la disminución de los impactos negativos de este tipo de actividades puede contribuir a la mejora del manejo integral de los EP.

La principal actividad de uso público en EP es el turismo. Por eso, en una primera instancia surgieron muchos documentos, guías y manuales que apuntaban a mejorar las prácticas de esta actividad. La "Cumbre de la Tierra" en Río de Janeiro (1992) o la Carta Mundial del Turismo Sostenible (Lanzarote, 1995) son importantes antecedentes que dieron notoriedad e importancia a esta cuestión.

A raíz de estas primeras iniciativas, distintos modelos y metodologías han intentado llevarse a cabo para lograr la relación adecuada entre el medio protegido y el resto de las potenciales actividades. Se pueden citar las Directrices sobre turismo en ecosistemas vulnerables (Convenio sobre la Diversidad Biológica); la Carta internacional sobre el turismo cultural: la gestión del turismo en sitios importantes para el patrimonio (Consejo Internacional de Monumentos y Sitios); la Declaración de Quebec sobre Ecoturismo; El turismo sostenible en las áreas protegidas: directrices para la planificación y gestión (Comisión Mundial de áreas Protegidas de la UICN); el Código mundial de ética para el turismo (Organización Mundial del turismo) y la Carta Europea del Turismo Sostenible.

El objetivo de todas estas propuestas era ordenar y planificar las actividades y lograr que cada reserva tenga un plan específicamente diseñado para tal fin. En Argentina la Administración de Parques Nacionales (APN) creó un documento en el cual se establecen lineamientos generales para el desarrollo del uso público en las unidades de conservación (BALABUSIC et al., 2003), del que se destacan algunas acciones que se promueven:

- Desarrollo del turismo sustentable y de oportunidades de trabajo asociadas a diferentes servicios de la actividad turística.

- Los mecanismos de cooperación con universidades u otras organizaciones afines, en temas relacionados con la planificación, desarrollo e implementación de las actividades en los Parques Nacionales.

- La elaboración de los planes de uso público para cada área protegida en el marco de los planes de manejo, como instancia previa a la ejecución de las acciones relacionadas con la actividad turística. 
METODOLOGÍAS DE PLANIFICACIÓN...

- El desarrollo de proyectos turísticos y otras alternativas productivas que sean compatibles con los objetivos de conservación, a partir de los criterios de manejo y uso asignados a las diferentes categorías y zonificación de los EP.

A pesar de la existencia de este documento, la Auditoría general de la Nación (Argentina) concluyó que del total de los EP de APN sólo el 13\% posee un plan de uso público aprobado. Además, en algunos de los Parques objeto de esta evaluación, ni siquiera se estudia el turismo y tampoco están preparados para recibirlo (AUDITORIA GENERAL DE LA NACIÓN, 2010).

Este ejemplo simplemente pone de manifiesto que planificar y gestionar las actividades de uso público no es tarea sencilla y encontrar la estrategia más adecuada para cada caso es un gran desafío. En parte, la razón de la proliferación de documentos como los citados previamente es que todavía existen muchos problemas e impactos negativos en los EP asociados al uso.

Esto no significa que no se haya conseguido ningún resultado, por el contrario, existe una mayor toma de conciencia y muchas prácticas se han modificado o adaptado como resultado de los intentos de reordenar las actividades. Es destacable que si bien estas primeras iniciativas estuvieron vinculadas al turismo, experiencias posteriores se han desarrollado en relación a todo tipo de actividades presentes en los EP.

Pero ¿cuál es la mejor alternativa para planificar el uso público? ¿Es la planificación de las actividades específicas, como el turismo? ¿Es la zonificación? ¿Es el ordenamiento del territorio a escalas regionales? O ¿es la búsqueda de nuevas metodologías integrales de planificación del uso público y la conservación? Las búsquedas han sido diversas y existen propuestas interesantes que podrían encuadrarse dentro de las distintas tendencias que se desprenden de los interrogantes planteados. Algunas de las experiencias más destacadas pueden agruparse dentro de las categorías presentadas a continuación.

\subsection{MÉTODOS ASOCIADOS A LAS EVALUACIONES DE EFECTIVIDAD Y LA UTILIZACIÓN DE INDICADORES}

Un primer grupo de metodologías lo constituyen aquellas derivadas de los resultados de las evaluaciones sobre la efectividad de la gestión y del manejo de EP. Estos documentos elaborados por los principales organismos de conservación de la naturaleza a nivel mundial y por las administraciones de muchos países, implementaron metodologías basadas en la utilización de indicadores e índices para identificar las principales problemáticas y deficiencias del sistema de EP.

Como se mencionó en el apartado anterior, el objetivo de estos documentos no era planificar el uso público, pero sí puede afirmarse que los resultados obtenidos evidenciaron que muchas problemáticas estaban relacionadas a las incompatibilidades de uso y a la falta de una adecuada planificación y gestión de las actividades. A raíz de esto, documentos complementarios han desarrollado una estrategia de planificación del uso público basada en subsanar y resolver los problemas identificados por las evaluaciones; algunas de ellas incluso utilizando parte de los indicadores propuestos en esos documentos.

Dentro de este grupo pueden incluirse numerosas experiencias, sobre todo la de algunos planes de manejo elaborados recientemente, la guía metodológica para la planificación del uso público en Argentina o la propuesta de "Evaluación del uso público en la red de parques nacionales de España" (SANTOS et al., 2012). Este último documento es un caso particular ya que propone una metodología de evaluación de la calidad del uso público, que a partir de sus resultados permite identificar lineamientos de planificación. La aplicación de la metodología propone evaluar distintos aspectos influyentes en el uso público, como los accesos, la zonificación, los programas de interpretación, de educación ambiental, de gestión del visitante, aspectos económicos, entre otros factores.

Los resultados de la aplicación de esta evaluación en algunos parques seleccionados permiten realizar recomendaciones para cada uno de los aspectos estudiados y en conjunto conforman las bases para lograr la planificación y la gestión del uso público (SANTOS et al., 2012). 
Otro caso que merece ser destacado son las "Directrices sobre diversidad biológica y desarrollo del turismo" (SECRETARÍA DEL CONVENIO SOBRE LA DIVERSIDAD BIOLÓGICA, 2004). Este trabajo tiene una visión diferente al anterior y analiza los impactos del turismo relacionados con el medio ambiente y con la diversidad biológica. Las directrices tienen un enfoque hacia la gestión del impacto como estrategia para planificar la actividad. Dentro de las propuestas realizadas pueden incluirse, entre otras medidas, lo relativo al emplazamiento del desarrollo y actividades turísticas, incluyendo el establecimiento de actividades adecuadas en distintas zonas designadas.

La iniciativa consiste en diferenciar entre los impactos de diversos tipos de turismo y medidas para controlar las corrientes turísticas en los destinos turísticos y en sus alrededores, así como en emplazamientos importantes, para promover la conducta adecuada de los turistas. El objetivo final es establecer límites en cuanto al número de visitantes, reducir a un mínimo los impactos y mantenerlos dentro de los límites de cambio aceptable en cualquier emplazamiento (SECRETARÍA DEL CONVENIO SOBRE LA DIVERSIDAD BIOLÓGICA, 2004).

Los ejemplos citados son experiencias destacadas, pero este tipo métodos quizás sean los más utilizados y los que mayor concentración de experiencias reúnan. La toma de decisiones sobre la gestión del uso público suele estar asociada a la corrección de los impactos y externalidades que genera. Es habitual en los planes de manejo considerar a las distintas actividades como amenazas para la conservación de los valores naturales y gestionarlas a partir de medidas de corrección o de prohibición de acuerdo a las distintas zonas.

\subsection{MÉTODOS ASOCIADOS AL ORDENAMIENTO Y LA PLANIFICACIÓN TERRITORIAL}

Las metodologías de planificación del turismo en EP y las propuestas de elaboración de planes de uso público tienen un fuerte basamento en el territorio y en la territorialidad de las actividades que se realizan. Este tipo de propuestas se articulan sobre la base de tres ejes principales: la identificación de atractivos o zonas de interés de acuerdo a la actividad que se trate, la conectividad entre los distintos puntos, y los flujos que se crean a partir de ellos.

En general se trata de iniciativas muy vinculadas al concepto de ordenamiento territorial y a algunos principios de la planificación ambiental. La definición de unidades ambientales o unidades territoriales, y la creación de modelos del territorio basados en diagnósticos previos son frecuentes en este tipo de propuestas, y suelen articularse con la utilización de cartografía temática y la implementación de sistemas de información geográfica (SIG).

Dentro de esta línea puede mencionarse en España la propuesta de "Planificación territorial del turismo" de ANTÓN CLAVE Y GONZÁLEZ REVERTÉ (2011). Si bien se refiere a una actividad específica como el turismo y no está vinculada únicamente a espacios naturales o áreas protegidas, su propuesta metodológica relacionada al territorio la vuelve muy a fin a la planificación de este tipo de espacios.

Se parte desde un diagnóstico territorial que incluye un análisis de los atractivos, los recursos, los agentes intervinientes y sus relaciones, para posteriormente definir un modelo territorial turístico. Se propone que este modelo territorial responda al paradigma escenario-mercado-producto y se elabore mediante la delimitación de unidades ambientales turísticas, en torno a las cuales se desarrollará efectivamente la actividad (ANTÓN CLAVÉ et al., 2011). Lo valioso de esta propuesta es como puede ser implementada y aplicada para otro tipo de actividades económicas con fuerte basamento territorial.

Siguiendo con el caso español "El plan de acción para los espacios naturales protegidos del Estado español" como otros documentos de la federación EUROPARC también se erigen dentro de este grupo de metodologías. Este documento proporciona elementos clave para la planificación y gestión de los espacios protegidos y recomienda para los planes de uso público un abordaje dentro del esquema de la ordenación del territorio. Se recomienda estructurar la planificación según las siguientes etapas: 
METODOLOGÍAS DE PLANIFICACIÓN...

- Diagnóstico de la situación de partida: marco legal, análisis de recursos, análisis de demanda, de agentes implicados, capacidad de acogida, análisis de impactos.

- Diseño de la planificación del uso público: programas de recibimiento, de actividades, de gestión de impactos, de educación, de formación.

- Administración del uso público: fórmulas de prestación de uso público, elaboración de modelos de pliegos, cooperación y administración con otras administraciones.

- Evaluación y seguimiento de la planificación del uso público.

Específicamente refiriéndonos a la gestión del turismo y la búsqueda de la sustentabilidad, se han diseñado marcas de calidad como Ecolabel o "Q de calidad", pero pareciera que el instrumento que más éxito y aceptación ha tenido es la Carta Europea del Turismo Sostenible (CETS). Esta carta estimula una planificación estratégica del turismo sostenible dentro de los EP involucrando a tres agentes fundamentales: el espacio protegido, las empresas turísticas dentro de estos espacios y los mayoristas de turismo.

La CETS puede ser considerada como una metodología de planificación del turismo porque su adopción implica el seguimiento de principios y lineamientos y el cumplimiento de ciertos compromisos por parte de los actores involucrados. La metodología a seguir incluye los siguientes aspectos:

- Una definición de la zona que reciba la influencia de la estrategia. Esta zona se puede extender más allá del espacio protegido.

- Una evaluación del patrimonio natural, histórico y cultural de la zona, la infraestructura turística y la situación económica y social, teniendo en cuenta aspectos como la capacidad, las necesidades y las oportunidades potenciales.

- Una evaluación de los visitantes actuales y de futuros mercados potenciales.

- Un conjunto de objetivos estratégicos para el desarrollo y la gestión del turismo, que incluyan:

- la conservación y mejora del medio ambiente y el patrimonio;

- el desarrollo económico y social;

- la protección y mejora de la calidad de vida de los residentes locales;

- la gestión de los visitantes y la mejora de la calidad de la oferta turística.

- Una descripción de los recursos disponibles y las partes relacionadas para la aplicación de la estrategia, la distribución de tareas y el establecimiento del orden de prioridades.

- Propuestas para el seguimiento de los resultados (EUROPARC FEDERATION, 2007)

Experiencias vinculadas a la planificación y ordenamiento territorial también pueden ser encontradas fuera de Europa. En México se ha desarrollado una escuela de estudios del turismo a partir del concepto de estructura territorial. Esta noción tomada de la escuela soviética de Geografía se refiere a la identificación de los elementos implantados en el espacio organizado en función de lugares de producción, lugares de consumo y sus interconexiones físicas y funcionales (SÁNCHEZ CRISPÍN et al., 2012).

Este concepto puede ser aplicado a cualquier actividad económica y así es como surgen los estudios de estructura territorial del turismo. El sentido principal de estos estudios es entender la funcionalidad de la actividad turística como sistema territorial para poder establecer su planificación y gestión. Sustentados en está construcción teórica se han llevado a cabo numerosos estudios a lo largo del territorio mexicano, pero también de muchos otros países de centro américa como Costa Rica, Guatemala, Panamá o Nicaragua (SÁNCHEZ CRISPÍN et al., 2005; SÁNCHEZ CRISPÍN et al., 2008; SÁNCHEZ CRISPÍN et al., 2012).

Otro caso destacado también implementado en México está referido a la ejecución de un modelo de predicción en los cambios en los usos del suelo debido al desarrollo de agricultura tradicional y ecoturismo (GARCÍA-FRAPOLLI et al., 2007). La metodología consiste en un análisis 
geohistórico de diversos factores que permiten predecir los cambios en el uso del suelo a partir de los cuales puede planificarse el uso público.

Este último ejemplo responde a un segmento de metodologías que intentan ordenar el territorio de los EP a través de mecanismos que ayudan a la toma de decisiones. Algunos de ellos se concentran en una dimensión específica como puede ser la movilidad dentro de estos espacios (IBEASA et al., 2014), o el análisis de las relaciones de compatibilidad entre distintas actividades como pueden ser el turismo y la pesca dentro de EP, pero también referidas a la agricultura, la ganadería y los usos urbanos (LOPES et al., 2015).

En otros casos las perspectivas son más generales y se busca ordenar el uso público con la utilización de metodologías para el diseño integral de los espacios protegidos desde la combinación de aspectos naturales y sociales para mejorar la toma de decisiones (CHEN et al., 2015; RUIZFRAU et al., 2015). En el primer caso la búsqueda es fomentar modelos de toma de decisiones acerca de las zonas de implantación de actividades productivas, mientras que el segundo propone una metodología para el diseño integral de EP a partir de la articulación entre la mirada científica y el trabajo con stakeholders.

No obstante las diferencias en el enfoque de cada uno de los estudios, todos ellos comparten la idea de proponer metodologías para establecer un reordenamiento o reorganización de los EP a partir del abordaje del uso público. La predilección por abordar la problemática desde la actividad turística es nuevamente notoria, pero no exclusiva, y en muchos casos sólo constituye una arista dentro de un esquema más amplio.

\subsection{MÉTODOS ASOCIADOS A LA ZONIFICACIÓN}

La zonificación es uno de los componentes básicos de todo EP. Como se explicó previamente, es la herramienta de diseño fundamental que permite diferenciar los distintos espacios dentro de una reserva y pretende establecer un ordenamiento de los usos y actividades que allí se desarrollan.

Pero la dificultad de conseguir este último objetivo, y la identificación de incompatibilidades y disfuncionalidades entre las zonificaciones establecidas y el uso real del espacio han llevado a desarrollar nuevos métodos para optimizar la funcionalidad de esta herramienta. Funcionalidad que depende del establecimiento de zonificaciones no sólo orientadas a la conservación sino también hacia el uso público. Los estudios que pueden encuadrarse dentro de esta orientación proponen metodologías de planificación del uso público integradas a los procesos de zonificación, independientemente de las técnicas y los procedimientos específicos que cada experiencia requiera.

En Italia, SENES Y TOCCOLINI (1998) propusieron una metodología de planificación del uso público en EP rurales denominada Ultimate Environmental Threshold. Esta propuesta se compone de una primera fase de identificación y evaluación de los recursos territoriales (tanto naturales como humanos), una segunda fase de definición de los distintos usos que se desarrollan en el área, con el subsecuente análisis de las interrelaciones entre recursos y actividades; y una tercera fase de identificación de las posibles zonas para la expansión de las actividades. Este esquema permite definir criterios para el uso del suelo, pero también prever la posible expansión de las actividades y reevaluar la zonificación en base a estos análisis. De esta forma se busca evitar futuras incompatibilidades y mejorar la funcionalidad general.

Otra experiencia desarrollada en Italia es una propuesta de zonificación para la conservación y el uso basada en un análisis multicriterio (GENELETTI et al., 2008). Esta metodología utiliza una combinación de índices para reevaluar la zonificación, divididos en factores ambientales, socioeconómicos y turístico-recreativos. Identifica zonas adecuadas para distintos usos, zonas de conflicto y zonas que pueden combinar distintos niveles de protección. El objetivo final es proteger valores naturales y culturales pero a la vez determinar cómo las facilidades turísticas se insertan adecuadamente dentro del esquema de zonificación.

Otro caso destacable fue experimentado en China donde se llevó a cabo una metodología de evaluación de la eficacia de la zonificación en EP (HULL et al., 2011). La metodología se 
METODOLOGÍAS DE PLANIFICACIÓN...

desarrolla a partir de un SIG que permite superponer capas de la zonificación vigente en cada parque, con capas de usos humanos y usos animales. Mediante la realización de trabajos de campo se analiza la distribución de urbanizaciones, facilidades turísticas y caminos entre las distintas zonas del parque y se propone una posible corrección de la zonificación a partir de la identificación de áreas estratégicas desfasadas con el uso sugerido en la zonificación actual.

Otro ejemplo relacionado, es el desarrollo de una herramienta para la facilitación de la toma de decisiones en cuestiones de zonificación y manejo de áreas costero-marinas (ÁLVAREZBERASTEGUI et al., 2014). En este caso también se utilizan indicadores e índices para elaborar tres tipos de mapas: mapas de calidad ambiental terrestre, de calidad ambiental marina y de presión antrópica. Cruzando los datos de los índices de cada mapa se pueden identificar zonas críticas que sirven para proponer correcciones de planificación y reordenar el uso público dentro de las reservas. En este caso por el tipo de reserva se destaca mucho su aplicación a actividades como la pesca, la acuicultura y el desarrollo de energías alternativas.

No todas las experiencias referidas surgen con el objetivo principal de planificar el uso público, pero se observa que a pesar de la diversidad de los objetivos, existe una búsqueda común hacia la integración de actividades de uso y conservación mediante la propuesta de nuevos métodos de zonificación.

\section{PROPUESTA METODOLÓGICA.}

El análisis sobre las distintas propuestas de planificación, evaluación y gestión del uso público llevadas a cabo en distintos países, nos acerca a las realidades y a las dificultades a las que se enfrentan distintos tipos de EP. Nos permite también realizar un trabajo de comparación entre los diferentes enfoques y abordajes utilizados e identificar las necesidades evidenciadas en cada caso. Las distintas visiones y aportes de las experiencias relevadas contribuyen a la construcción de una perspectiva metodológica sustentada en base a una serie de componentes clave, cuyo desarrollo y ejecución deberá ser adaptado a las particularidades de cada caso. A partir de esta concepción, se propone a continuación (Figura 1) un esquema metodológico básico para la planificación del uso público, articulado sobre los ejes que se consideran fundamentales para abordar la problemática del uso del espacio en los EP.

La Figura 1 presenta una propuesta metodológica para la planificación del uso público enmarcada dentro de un enfoque próximo a las teorías del ordenamiento territorial y a la concepción ampliada y actual de este concepto. Se entiende que los EP constituyen sistemas territoriales complejos que persiguen objetivos específicos y para los que necesitan un ordenamiento del territorio determinado.

Las especificidades de este tipo de espacios, requieren la consideración de ciertas cuestiones que en otro tipo de propuestas de ordenación territorial pueden ser abordadas de manera distinta. El esquema elaborado en este caso toma los principales ejes temáticos observados en los estudios analizados, los combina con técnicas concretas y los articula de forma que puedan constituirse en una propuesta metodológica básica.

Esta propuesta metodológica comprende cinco momentos o etapas fundamentales, que requieren de la articulación de una serie tareas y la consecución de objetivos concretos. El origen del proceso se sustenta en los principios del ordenamiento territorial y en las metodologías de evaluación de EP. Se busca en un primer momento analizar los criterios de planificación y ordenamiento del territorio que afectan a un determinado espacio. Dentro de esta etapa, la identificación de planes, de códigos de ordenamiento territorial, y de toda la normativa vigente, es de significativa importancia. El objetivo principal será el de conocer en detalle los condicionantes para la gestión del territorio, la caracterización del entramado jurídico-administrativo, la identificación de las competencias y responsabilidades sobre el territorio; y esencialmente recopilar información de base para los posteriores análisis. 
FIGURA 1

Propuesta metodológica para la planificación del uso público

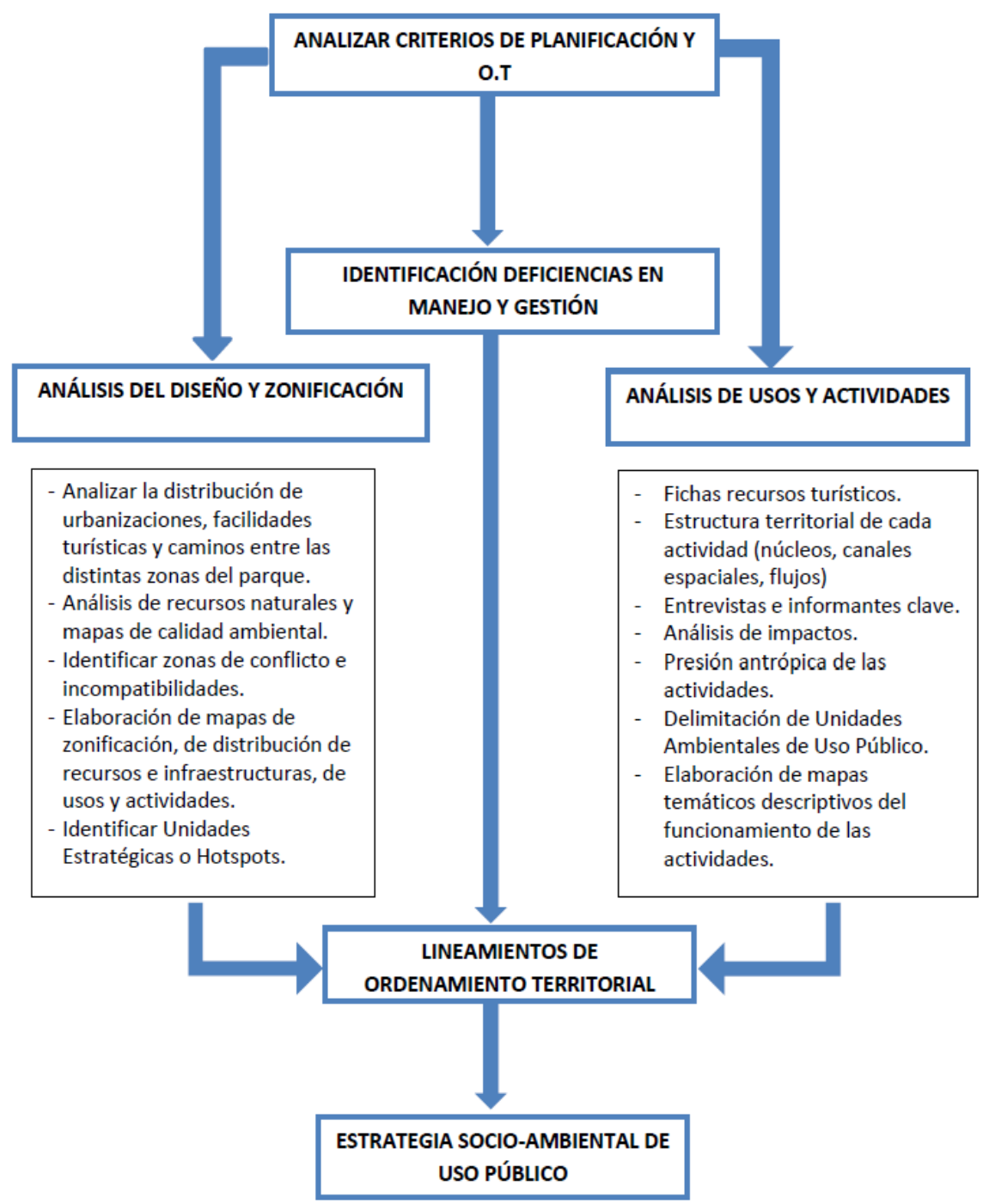

Fuente: Elaboración propia

El segundo momento consiste en la identificación de problemáticas de manejo y gestión del espacio. Este segundo momento se produce a partir de la revisión documental y bibliográfica iniciada en la etapa anterior, pero se complementa necesariamente con trabajos de reconocimiento del territorio y con la aplicación de entrevistas, talleres de consulta y cuestionarios a responsables de la gestión, a informantes clave, a la comunidad local y a usuarios del lugar. La problemática territorial actual nos presenta el compromiso de generar iniciativas reales y democráticas de participación ciudadana para conocer los intereses y voluntades de los distintos sectores; para acercarnos a las realidades del territorio; y para manejar los conflictos sociales latentes en cada espacio. En esta etapa la implementación de las técnicas mencionadas, entre otras, deberán apuntar a obtener este tipo de información tan indispensable para establecer un ordenamiento del territorio.

Las dos primeras etapas conforman un diagnóstico orientado del EP, en donde no solamente se ponen de manifiesto los impactos negativos presentes o sus deficiencias, sino que busca entender el contexto en el cual se encuentran inmersos estos espacios y caracterizar sus problemáticas desde la interpretación de los EP como sistemas territoriales complejos. 
METODOLOGÍAS DE PLANIFICACIÓN...

El tercer momento consiste en realizar un análisis y evaluación detallados de dos elementos clave en estos espacios: el diseño y zonificación; y los usos y actividades. El objetivo de esta fase es comprender la estructura territorial del EP, identificar usos actuales y potenciales, intereses de las comunidades locales, formas de apropiación del espacio y todo lo relativo a la dinámica integral del EP como sistema territorial. Como se observa en la Figura 1, para cumplimentar con esta tarea será necesaria la combinación y articulación de variadas técnicas y análisis que deberán seleccionarse de acuerdo al tipo de espacio. El dialogo y la participación ciudadana se tornan nuevamente indispensables a la hora de entender la dinámica del espacio analizado.

Para abordar la cuestión del diseño (tamaño, forma, límites) y la zonificación se debe considerar la información recabada en la primera etapa, referida al marco legal o jurídico-administrativo que establece condicionantes sobre el territorio estudiado. Pero a su vez, en la propuesta realizada se incluyen ciertos análisis y tareas indispensables para complementar la evaluación de estos aspectos. El relevamiento de urbanizaciones, la localización de infraestructuras, la identificación de las distintas zonas, de las áreas conflictivas o de las áreas estratégicas para cada uso, se consideran trabajos imprescindibles. La elaboración de cartografía con la distribución de recursos, de usos y actividades, y de unidades estratégicas ayudaran a una reevaluación de la funcionalidad de la zonificación vigente y del diseño en su conjunto.

En el análisis de las actividades el objetivo será en primera instancia, identificar tanto aquellas presentes como las potenciales. Pero más importante aún, es lograr una comprensión cabal de las características y el funcionamiento de cada una de ellas y las relaciones de múltiple influencia que se generan, en especial en términos de compatibilidad entre ellas. En este caso la combinación de técnicas y procedimientos dependerá de la complejidad del espacio objeto de estudio.

Para cada una de las actividades se propone entender su estructura -articulada en núcleos centrales, canales de relación y flujos que se forman a partir de ella-, pero también es indispensable dimensionar los impactos y la presión que generan. Como complemento, la utilización y elaboración de mapas temáticos puede contribuir a explicar el funcionamiento de las actividades y delimitar así unidades ambientales de uso público. El objetivo final de la contrastación de los análisis realizados respecto al diseño, la zonificación y las actividades, será comprender cuál es la dinámica real del espacio y encontrar disfuncionalidades entre los objetivos de conservación y el uso público, y entre la zonificación y el uso efectivo que se le da al espacio.

Los resultados de estas tres etapas permitirán establecer, en un cuarto momento, nuevos lineamientos generales para reordenar la unidad de conservación analizada. Estos lineamientos pueden estar dirigidos esencialmente hacia el interior del EP, proponiendo correcciones en la zonificación, modificaciones o prohibiciones en el desarrollo de alguna actividad, o sugiriendo cambios en determinadas estrategias de gestión. De ser necesario los lineamientos podrán estar orientados hacia el entorno del EP o referidas al marco legal. Dentro de este grupo de propuestas pueden incluirse modificaciones de tamaño y límites; referidas a la conformación y las competencias de los organismos de gestión; dirigidas hacia la normativa que los regula; o respecto a la articulación con otros espacios de dominio público o privado que puedan tener influencia sobre el caso analizado.

La última etapa de este proceso propone el desafío de conformar una nueva estrategia territorial para el EP elegido. Esta estrategia territorial es denominada "socio-ambiental de uso público" porque pretende conjugar en una misma propuesta, formas de utilización del espacio que equilibren factores sociales y ambientales. Equilibrio que debe conseguirse como consecuencia de los análisis territoriales realizados, de la información recabada sobre los usos y actividades, y particularmente de la correcta articulación y compatibilización de los intereses sociales detectados.

La estrategia debe considera el contexto actual de competencia por el espacio, y la detectada necesidad de propiciar EP cada vez más vinculados con el resto de las actividades e intereses de la sociedad. La innovación estará dada entonces, por centrar la mirada en la regulación de las formas de utilización del espacio tanto para su conservación como para el uso. De esta forma, el espacio es visto como un todo integrado, en donde los factores sociales y los referidos al uso público, 
adquieren mayor protagonismo respecto a otro tipo de propuestas, buscando una mejor articulación con los factores ambientales y los objetivos de conservación.

Lo que se pretende es cambiar la lógica tradicionalmente establecida respecto al diseño de EP, en donde las actividades de uso público se insertan en la dinámica de cada reserva de forma desarticulada con las estrategias de conservación; en un proceso o etapa posterior, y en muchos casos sin una adecuada planificación. El objetivo de máxima de pensar una estrategia socioambiental de uso público, será romper con esta dinámica, rediseñar el EP y propiciar una gestión integrada del espacio, que considere de forma equilibrada todas las alternativas de uso presentes, y posibles.

\section{PERSPECTIVAS DE PLANIFICACIÓN DEL USO PÚBLICO}

Las distintas iniciativas analizadas y mencionadas respecto al manejo del uso público en EP sólo representan algunas experiencias dentro de las tendencias más destacadas de los últimos años. Detrás de cada uno de los grupos conformados y de cada propuesta individualmente, podrían citarse cientos de documentos y experiencias que escapan a las posibilidades de este trabajo.

Los resultados de este trabajo permiten establecer algunas discusiones respecto a las perspectivas futuras en la planificación del uso público. En primera instancia se denota un creciente interés por atender las cuestiones relacionadas con los usos y actividades dentro de los espacios protegidos. Específicamente, se aborda esta cuestión a partir de la búsqueda y la propuesta de nuevas metodologías y estrategias para planificar y gestionar los distintos usos que tienen lugar en estos espacios.

A pesar de la diversidad de enfoques con que esta problemática es abordada, la motivación principal de la mayor parte de los estudios es la búsqueda de soluciones y alternativas para disminuir las disfuncionalidades entre las actividades de conservación y otro tipo de usos. Se evidencia también que esta motivación surge de lo que pareciera ser un consenso generalizado acerca de la necesidad de ocuparse de la planificación del uso público como estrategia indispensable para la consecución de los objetivos de conservación de las EP, y de los sistemas de espacios protegidos.

Por otro lado, aunque se registran numerosas propuestas abocadas a la resolución de problemáticas sectoriales, o a la planificación y gestión de actividades específicas como puede ser el turismo o la pesca; se observa una preferencia hacia los abordajes desde visiones integrales de la problemática, sustentadas en los principios del ordenamiento territorial. Incluso en aquellos estudios que realizan propuestas concretas y específicas se reconoce que su verdadero valor y utilidad vendrá dado si se realizan en el marco de un abordaje complejo y articulado.

Las diversas experiencias analizadas nos permiten observar que el futuro de la planificación del uso público está estrechamente vinculado con el desarrollo de metodologías sólidas e innovadoras. Más allá de las especificidades de cada uno de los estudios citados, todos ellos intentan proponer nuevos métodos y técnicas para generar un cambio en la forma en que los usos y actividades son planificados y gestionados dentro de cada unidad de conservación. Podría decirse que estos estudios coinciden en entender que una de las claves del éxito para la correcta articulación del binomio usoconservación reside en establecer nuevas metodologías de planificación.

No puede desconocerse igualmente, que cada grupo de metodologías propone una estrategia que centra su interés en determinadas cuestiones, como las vinculadas al ordenamiento y planificación territorial, las que ponderan la zonificación como herramienta, las que se concentran en la planificación de actividades específicas, o las que se preocupan por evaluar la calidad y la efectividad. Sin embargo, en general se destaca la necesidad de abordajes integrales y se entiende el rol de cada iniciativa en el marco de un sistema complejo de relaciones.

Las perspectivas de planificación del uso público se reflejan en la visión de este trabajo a través del esquema presentado en la Figura 1. Cada uno de las etapas representa una línea de acción o un eje central a tener en cuenta en los procesos de planificación actuales o futuros. Independientemente 
del orden asignado en este caso, se entiende que el análisis de estos ejes y líneas de acción estará presente en gran parte de las iniciativas futuras.

La detectada necesidad de abordajes integrales y de enfoques participativos se representa a través de la concepción del ordenamiento territorial como elemento transversal de todo el proceso. La identificación de deficiencias en el manejo y la gestión se constituye como una etapa fundamental en los procesos de planificación que buscan establecer diagnósticos iniciales. Esta labor ya está muy presente en los documentos de evaluaciones desarrollados en los últimos años, pero se considera que seguirá siendo de gran importancia para la realización de las posteriores etapas de índole prospectivas.

Por su parte, la tercera etapa planteada en la Figura 1 quizás represente el factor principal a tener en cuenta en la planificación del uso público futura. Los análisis sobre la eficacia de las zonificaciones y sobre la adecuación de los diseños de los EP para la consecución de los objetivos de cada unidad de conservación han sido muy discutidos en los últimos años y representan una tendencia en varios países. A su vez, la preocupación por la gestión de las distintas actividades, usos urbanos y productivos que tienen lugar en estos espacios, también se ha incrementado en los últimos tiempos. Puede observarse que el estudio y la búsqueda de estrategias de manejo de estos factores se constituyen como una clara perspectiva a futuro en los procesos de planificación y gestión.

Las últimas dos etapas propuestas en el esquema metodológico se condicen con dos de los principales desafíos y necesidades de la gestión territorial en la actualidad: el manejo y reducción de la conflictividad territorial; y la articulación del binomio uso-conservación. La conflictividad derivada de la competencia y de las disputas por el uso del espacio es una problemática extensible a muchos territorios, pero especialmente se manifiesta cuando las alternativas que se manejan y los intereses que están en juego son muy diferentes o tienen un alto grado de incompatibilidad. Este es el caso de los EP donde esta relación se manifiesta, a grandes rasgos, a través de las alternativas de conservación contra las de uso.

Siguiendo con las etapas establecidas en la Figura 1, tanto en la definición de lineamientos de ordenamiento territorial y en la creación de una estrategia socio-ambiental de uso público subyace la idea de buscar alternativas para afrontar los dos desafíos mencionados en las líneas anteriores. La definición de los lineamientos de ordenamiento territorial y el diseño de una estrategia para el uso público, desarrollados en un contexto participativo y democrático, pueden contribuir a subsanar los conflictos de intereses entre los distintos actores sociales y pueden establecer regulaciones claras y concisas acerca de las formas de utilización del espacio permitidas, y respecto a la adecuada articulación entre las distintas alternativas seleccionadas.

\section{CONCLUSIONES}

El trabajo desarrollado permite arribar a ciertas conclusiones respecto a la evolución de las metodologías de planificación del uso público. En primer lugar la comparación de distintos casos de estudio y el abordaje de documentos elaborados por organismos internacionales ha permitido analizar la evolución de esta problemática. Este primer análisis ha evidenciado un creciente interés por planificar el uso público dentro de los EP y por implementar nuevas estrategias para mejorar su manejo y reducir las externalidades y los impactos negativos derivados.

Ha sido justamente esta motivación por minimizar impactos, la que ha impulsado una preponderancia de estudios desarrollados a partir del diseño de metodologías de evaluación, buscando establecer diagnósticos para posteriormente establecer estrategias de manejo. Si bien este tipo de estudios no han resuelto íntegramente los problemas manifiestos, parte de las técnicas y metodologías aplicadas tienen mucha influencia actualmente en los procesos de planificación, y es probable que continúen teniéndola en el corto plazo.

No obstante, los distintos casos de estudio observados nos permiten también afirmar, que existe una muy variada búsqueda por encontrar alternativas de planificación y manejo del uso público.

Papeles de Geografía, 62 (2016), 119-136 
Esta búsqueda ha estado dirigida hacia la planificación de actividades específicas y se ha centrado fundamentalmente en cuestiones como la actualización y reevaluación de zonificaciones y diseños y las disfuncionalidades que existen entre ellos y el uso del espacio. Esta situación pone de manifiesto la necesidad de desarrollar nuevos abordajes que permitan abarcar la totalidad de los factores y aspectos que deben considerarse.

La propuesta metodológica realizada en este trabajo puede decirse que es una construcción, o un producto, derivado del análisis de numerosas experiencias de los últimos años. Este trabajo permitió identificar tanto patrones y criterios comunes, como ciertas carencias y deficiencias. En ese sentido, el esquema metodológico propuesto representa una alternativa que combina lo más destacado de los métodos y técnicas de las propuestas sectoriales, y los encuadra dentro de los principios integrales del ordenamiento territorial.

El valor de la propuesta -más allá de su posibilidad de aplicación a casos concretos- radica en poner de manifiesto la principal perspectiva de planificación del uso público, relacionada con la profundización de la búsqueda de estrategias socio-ambientales para la gestión de los EP. Estrategias en donde los diseños y las zonificaciones no sean pensados sólo a base de criterios físico-naturales y en donde la planificación de los usos y actividades no se realice de forma aislada y sin articularse con los objetivos de conservación.

Puede afirmarse también, que el esquema metodológico propuesto en este trabajo no se constituye como una metodología enteramente desarrollada. Se trata de un esquema básico que representa el orden lógico del proceso metodológico y articula cada una de las etapas de este proceso con las principales técnicas necesarias para la consecución de los objetivos implícitos.

En definitiva, este trabajo a través del repaso de distintas experiencias globales y a través de la configuración de una propuesta metodológica afín a las necesidades evidenciadas, intenta poner de manifiesto una visión sobre los EP. Visión que trata de pensar a los espacios protegidos como espacios sociales, en donde las necesidades naturales y humanas sean articuladas de forma tal, que permitan su planificación conjunta, en pos de optimizar las funciones y los objetivos con los cuales estos espacios fueron creados.

Restará a futuro, continuar evaluando la evolución de la planificación del uso público, la efectividad de las distintas técnicas y metodologías relevadas y la manifestación de las perspectivas identificadas. Pero especialmente, determinar la posibilidad de que sean los abordajes amparados en el concepto de ordenamiento territorial los que guíen estas nuevas visiones de los EP, que permitan armonizar la relación uso-conservación y propiciar un acercamiento hacia el alcance de los principales objetivos y metas de cada unidad de conservación.

\section{BIBLIOGRAFÍA}

ALMIRÓN, M.; BALABUSIC, A.; BOSSO, A.; BUKART, R.; CARMINATI, A.; CARPINETTI, B.; FOURCADE DE RUIZ, M.; GAZIBE, V.; HAENE, E.; LEPERA, G.; LUNAZZI, M.; MACHAIN, N.; MANZIONE, M.; MARTÍN, G.; MELHEM, S.; MENVIELLE, M.; MOLINARI, R.; MONGUILLOT, J.; MORENO, D.; OCHOA, M.; PAZ BARRETO, D.; RAFFO, L.; RODRIGUEZ, V.; SIMONETTI DE URIBELARREA D. y SOMMA, D. (2007): Las Áreas Protegidas en la Argentina. Herramienta Superior para la conservación de nuestro patrimonio natural y cultura. APN y FVSA, Buenos Aires, 83 pp.

ÁlVAREZ-BERASTEGUI, D., AMENGUAL, J., COLL, J., REÑONES, O., MORENO-NAVAS, J. y AGARDY, T. (2014): Journal for Nature Conservation. "Multidisciplinary rapid assessment of coastal areas as a tool for the design and management of marine protected areas", vol. $22 \mathrm{n}^{\circ} 1$, p. 1-14. doi:10.1016/j.jnc.2013.07.003

ANTÓN CLAVÉ, S., y GONZÁLEZ REVERTÉ, F. (2011): Planificación territorial del turismo. Barcelona, Editorial UOC, 216 pp.

AUDITORIA GENERAL DE LA NACIÓN. (2010): Examen del Plan de Gestión Institucional de la Administración de Parques Nacionales. AGN, Buenos Aires, 173 pp.

Papeles de Geografía, 62 (2016), 119-136 
METODOLOGÍAS DE PLANIFICACIÓN...

BALABUSIC, A.; CASELLI, R.; GALLARDO, A. y MELHEM, S. (2003): Metodología para planificar el uso público en Áreas Protegidas bajo jurisdicción de la Administración de Parques Nacionales. Administración de Parques Nacionales, Buenos Aires, 82 pp.

BURKART, R. (2005): La situación ambiental Argentina. "Las áreas protegidas de la Argentina". En Brown, A., Martínez Ortiz, U., Acerbi, M., y Corcuera, J. (Eds). Fundación Vida Silvestre, Buenos Aires, pp. 399-404.

CHEN, C.-T., LIN, H.-J., KO, T.-T., CHENG, H.-C., WRIGHT, J. R., y CHANG, Y.-C. (2015): Ocean \& Coastal Management. "Spatial resource allocation modeling for marine protected areas design: The case of Kaomei coastal wetland", vol. 110, pp. 46-56. doi:10.1016/j.ocecoaman.2015.03.007

DE LA MAZA ELVIRA, J., CADENA GONZÁLEZ, R., PIGUERÓN WIRZ, C., y SC, Q. C. E. (2003): Estado actual de las áreas naturales protegidas de América Latina y el Caribe. Lima, Quercus consultora económica S.C.

DOUROJEANNI, M. J. y QUIROGA, R. (2006): Gestión de áreas protegidas para la conservación de la biodiversidad: evidencias de Brasil, Honduras y Perú. Banco Interamericano de Desarrollo Washington, $122 \mathrm{PP}$.

ELBERS, J. (2011): Las áreas protegidas de América Latina: Situación actual y perspectivas para el futuro. UICN, Oficina Regional para América del Sur, Quito 227 pp.

EUROPARC. (2014): Anuario 2013 del estado de las áreas protegidas en España. Madrid, Fundación Fernando Gonzáles Bernáldez.

EUROPARC Federation. (2007): La Carta Europea del Turismo Sostenible en los espacios protegidos: texto oficial. Grafenau, Europarc.

GARCÍA-FRAPOLLI, E., AYALA-OROZCO, B., BONILLA-MOHENO, M., ESPADASMANRIQUE, C. y RAMOS-FERNÁNDEZ, G. (2007): Landscape and urban planning "Biodiversity conservation, traditional agriculture and ecotourism: Land cover/land use change projections for a natural protected area in the northeastern Yucatan Peninsula, Mexico", vol. 83, $n^{\circ}$ 2, p.137-153. doi:10.1016/j.landurbplan.2007.03.007

GENELETTI, D., y VAN DUREN, I. (2008): Landscape and Urban Planning "Protected area zoning for conservation and use: A combination of spatial multicriteria and multiobjective evaluation", vol. 85, $n^{\circ}$ 2, p. 97-110. doi:10.1016/j.landurbplan.2007.10.004

GIACCARDI, M., y TAGLIORETTE, A. (2007): Efectividad del manejo de las áreas protegidas marino costeras de la Argentina. Secretaría de Ambiente y Desarrollo Sustentable de la Nación, Fundación Patagonia Natural y Fundación Vida Silvestre Argentina, Buenos Aires, 108 pp.

GÓMEZ MENDOZA, J., y VALLE BUENESTADO, B. (1999): Geografía y espacios protegidos, Asociación de Geógrafos Españoles y Federación de Espacios Naturales protegidos de Andalucía. "Introducción al sistema de áreas protegidas de Argentina", p. 159-170.

GUDIÑO, M.E. (2010): Scripta Nova: revista electrónica de geografía y ciencias sociales. "Del urbanismo reglamentario a las nuevas concepciones de ordenamiento territorial", no. 14, pp. 25.

HULL et al. (2011): Biological Conservation. "Evaluating the efficacy of zoning designations for protected area management", vol. 144, $n^{\circ}$ 12, p. 3028-3037. doi:10.1016/j.biocon.2011.09.007

IBEASA, A., MOURAA, J. L., DELL'OlIOA, L., y ALONSO, B. (2014): Procedia-Social and Behavioral Sciences "XVIII Congreso Panamericano de Ingeniería de Tránsito, transporte y Logística", vol. 162, p. 1-5. doi:10.1016/j.sbspro.2014.12.179

ICOMOS. (1999): Carta Internacional sobre Turismo Cultural. La Gestión del Turismo en los sitios con Patrimonio Significativo. ICOMOS, México.

ISNARD, H. (1975): Annales de géographie. "L’espace du géographe", p. 174-187.

LOPES, P. F. M., PACHECO, S., CLAUZET, M., SILVANO, R. A. M., y BEGOSSI, A. (2015): Ecosystem Services. " Fisheries, tourism, and marine protected areas: Conflicting or synergistic interactions?", vol. 16, p. 333-340. doi:10.1016/j.ecoser.2014.12.003

MASSIRIS CABEZA, Á.M. (2002): Scripta Nova: revista electrónica de geografía y ciencias sociales, "Ordenación del territorio en América Latina", no. 6, pp. 125. 
MCDONALD, R. I., FORMAN, R. T., KAREIVA, P., NEUGARTEN, R., SALZER, D., y FISHER, J. (2009): Landscape and Urban Planning. "Urban effects, distance, and protected areas in an urbanizing world", vol. 93, $n^{\circ}$ 1, p. 63-75. doi:10.1016/j.landurbplan.2009.06.002

MCDONALD, R. I., KAREIVA, P., y FORMAN, R. T. (2008): Biological conservation. "The implications of current and future urbanization for global protected areas and biodiversity conservation", vol. 141, $n^{\circ}$ 6, p. 1695-1703. doi:10.1016/j.biocon.2008.04.025

MOREA, J. P. (2014): Revista Universitaria de Geografía. "Situación actual de la gestión de las áreas protegidas de la Argentina: Problemáticas actuales y tendencias futuras", vol. $23 n^{\circ} 1$, p. 57-75. Disponible en: http://www.scielo.org.ar/scielo.php?pid=S185242652014000100003\&script=sci_arttext\&tlng=en

OMT. (1999): Código Ético Mundial para el turismo. OMT, Santiago de Chile.

OMT. (2002): Turismo sostenible en áreas protegidas. Directrices de planificación y gestión. OMT y UICN, Madrid.

ONU. (1995): Carta del Turismo Sostenible, Conferencia Mundial de Turismo Sostenible. ONU, Lanzarote, España. Disponible en: www imacmexico.org

ONU. (2002): Declaración de Quebec sobre el Ecoturismo. ONU, Quebec. Disponible en: http:// www.cinu.org.mx/eventos/turismo2002/doctos/dec_quebec.htm

RUIZ-FRAU, A., POSSINGHAM, H. P., EDWARDS-JONES, G., KLEIN, C. J., SEGAN, D., y KAISER, M. J. (2015): Ocean \& Coastal Management. "A multidisciplinary approach in the design of marine protected areas: Integration of science and stakeholder based methods", $\mathrm{n}^{\circ}$. 103, p. 86-93. doi:10.1016/j.ocecoaman.2014.11.012

SÁNCHEZ CRISPÍN, Á., LÓPEZ LÓPEZ, Á., y PROPIN FREJOMIL, E. (2005): Investigaciones geográficas. "Estructura territorial del turismo en la Zona Metropolitana de Monterrey, México", $\mathrm{n}^{\circ} .58$, p. 80-105. Disponible en http://www.redalyc.org/articulo.oa?id=56905809

SÁNCHEZ CRISPÍN, Á., MOLLINEDO BELTRÁN, G., y PROPIN FREJOMIL, E. (2012): Investigaciones geográficas. "Estructura territorial del turismo en Guatemala", nº 78, p. 104121. Disponible en http://www.scielo.org.mx/scielo.php?script=sci_arttext\&pid=S018846112012000200009

SÁNCHEZ CRISPÍN, Á., URBINA BRAVO, M. Á., y PORPIN FREJOMIL, E. (2008): Cuadernos de Turismo. "Rasgos territoriales del turismo en la isla de Ometepe, Nicaragua", ${ }^{\circ} 21$, p. 159179. Disponible en http://revistas.um.es/turismo/article/view/25031

SANTOS, M. (1997). Metamorfosis del espacio habitado. Sao Paulo, Hucitec.

SANTOS, M. M., y DEL ÁLAMO, J. B. (2012): El uso público en la red de parques nacionales de España una propuesta de evaluación. Madrid, Organismo Autónomo Parques Nacionales.

SECRETARÍA DEL CONVENIO SOBRE LA DIVERSIDAD BIOLÓGICA. (2004): Directrices sobre Diversidad Biológica y Desarrollo del Turismo: Directrices internacionales para actividades relacionadas con el desarrollo del turismo sostenible en ecosistemas vulnerables, terrestres, marinos y costeros y hábitats de gran importancia para la diversidad biológica y áreas protegidas, incluidos los ecosistemas frágiles, ribereños y de montañas. Secretaría del Convenio sobre la Diversidad Biológica, Montreal, 34 pp.

SENES, G., y TOCCOLINI, A. (1998): Landscape and urban planning. "Sustainable land use planning in protected rural areas in Italy", vol. 41, $n^{\circ} 2$, p. 107-117. doi:10.1016/S0169$\underline{\text { 2046(97)00064-9 }}$ 